\title{
USO DE REALIDAD AUMENTADA COMO APOYO A UN SISTEMA DE NAVEGACIÓN EN NEUROCIRUGÍA
}

\author{
Pedro Luis Solarte Correa ${ }^{1}$, José María Sabater Navarro² ${ }^{2}$ Eliana Margarita Aguilar Larrarte ${ }^{1}$, Oscar Andrés \\ Vivas Albán ${ }^{1}$, José María Vicente Samper ${ }^{2}$ \\ ${ }^{1}$ Universidad del Cauca, Popayán-Colombia, ${ }^{2}$ Universidad Miguel Hernández, Elche-España \\ (plsolarte@unicauca.edu.co,j.sabater@umh.es, eaguilar@unicauca.edu.co, avivas@unicauca.edu.co,jose.vicentes@umh.es)
}

\begin{abstract}
Resumen
La cirugía mínimamente invasiva (MIS) como su nombre lo indica es una técnica quirúrgica que tiene como objetivo reducir los daños sobre los tejidos y órganos del paciente que puedan resultar del procedimiento médico como tal. Sin embargo, uno de los desafios de esta técnica radica en superar la limitación visual y en desarrollar una buena capacidad de crear imágenes mentales de las posibles estructuras anatómicas no visibles. En este artículo se presenta un prototipo de sistema de navegación neuroquirúrgica basada en la técnica de realidad aumentada (AR) soportada en la tecnología emergente de Head-Mounted Displays (HMD) para crear un escenario que genera una sensación visual ampliada de estructuras neurológicas creadas por computador a partir de imágenes de resonancia magnética (IMR) que permite acceder a estructuras anatómicas obtenidas a partir de datos médicos reales evitando el acceso fisico del paciente.
\end{abstract}

Se realizaron dos pruebas de interacción del sistema con diez sujetos de prueba concluyendo que existe un gran interés en el uso de la tecnología la cual fue usable, absorbente y presentó un nivel de inmersión aceptable.

Palabras clave: Realidad aumentada, neurocirugía, navegación quirúrgica.

\section{INTRODUCCIÓN}

La cirugía mínimamente invasiva actualmente representa la evolución de las técnicas quirúrgicas cuyo fin es brindar un mayor beneficio al paciente, aunque ésta aumenta la dificultad de su ejecución ya que la percepción de la profundidad se ve reducida en una gran medida, se limita el campo de visión y disminuye el sentido del tacto [10], [14]. Con la cirugía mínimamente invasiva de cabeza se introduce el concepto de navegación quirúrgica, la cual se hace más adecuada en zonas anatómicas ubicadas en los dos tercios superiores de la cabeza, como lo son la base del cráneo, orbita, el contorno craneal, fosas nasales, senos maxilares y la región preauricular [4]. En el campo de la cirugía mínimamente invasiva, la realidad aumentada (AR) representa una mejora para dicha técnica, ya que permite combinar la virtualidad con la realidad, ayudando a la interpretación de información que anteriormente no estaba disponible para el cirujano, y proporcionando datos importantes de imágenes tratadas por computador para el desarrollo de una intervención quirúrgica [8]. Actualmente para el cirujano, el análisis de imágenes por ultrasonido (USG), tomografía computarizada (CT), resonancia magnética (MRI) y otras técnicas de imagen, representa el método convencional para el diagnóstico y tratamiento de varios problemas de salud, el cual lo limita de datos importantes como la profundidad, ya que son técnicas que brindan información en 2D, además se ha demostrado que con la evolución del cerebro a menudo es tan tedioso limitar pensamiento a íconos y menús de una pantalla plana o una imagen [15], [9]. Es aquí donde la realidad aumentada (AR) mediante la construcción de los modelos 3D basados en dichas imágenes médicas, proveen un aporte a las MIS con un campo más completo de información en la cual pueda navegar en un modelo virtual tridimensional sin necesidad de acceder físicamente al paciente [2], [3]. Los avances en realidad aumentada representan nuevas modalidades en la práctica y la enseñanza de la neurocirugía, puesto que en esta es la única disciplina donde se ha estandarizado la AR ya que, en esta el cráneo sirve de referencia rígida. Aunque el cerebro no es rígido, su deformación es mínima (conocida como 'brain shift') y para fines de realidad aumentada se ha tomado como rígida [11], [12].

\section{MATERIALES Y MÉTODOS}

\subsection{META II / UNITY 3D}

El casco Meta II de Metavisión, es un dispositivo que permite al usuario utilizar el espacio que lo rodea para visualizar e interactuar con objetos 3D. En la actualidad el casco el Meta II, provee una excelente experiencia en un mundo 3D en el cual se pueden usar las manos, logrando que el usuario pueda tocar, mover y sujetar objetos virtuales de manera intuitiva [1]. Este 
casco es compatible con Unity 3D versión 2017.3.0f3 la cual fue utilizada para el desarrollo de este sistema. Unity 3D es un software para desarrollo de juegos en 2D y 3D, y entornos virtuales multiplataforma [7]. El SDK 2.7.0 de Meta 2 para Unity 3D brinda las herramientas necesarias para desarrollar aplicaciones de realidad aumentada en el casco.

\subsubsection{Obtención de la orientación de la vía de abordaje}

Los cuaterniones, o cuaternios de Hamilton, son una expresión matemática para representar orientaciones en un espacio 3D. Son de la forma (1) donde $a, b, c, d$ son números reales y las tres unidades imaginarias $i, j$, $k$ tienen como cuadrado igual a -1 como se muestra en (2), y se utilizan en este caso para representar la rotación de un objeto [13], [5].

$$
\begin{aligned}
& a+b i+c j+d k \\
& i^{2}=j^{2}=k^{2}=-1
\end{aligned}
$$

En Unity 3D cada objeto posee un transform, el cual es el componente que define su posición, rotación y escala en una escena. La rotación de ese transform es almacenada en una variable de tipo Quaternion, que funciona de manera similar a los vectores, pero con la diferencia que posee cuatro componentes independientes (X, Y, Z y W) y funcionan en conjunto para definir cualquier rotación que un objeto pueda necesitar. Unity 3D convierte los cuaterniones en ángulos de Euler para su visualización en el transform del objeto, pero internamente no trabaja con estos ya que los ángulos de Euler están sujetos al fenómeno conocido como Gimbal Lock el cual consiste en que en algunas secuencias de rotación es posible que dos de estos tres ángulos provoquen una rotación alrededor del mismo eje del objeto, bloqueando uno de los giros [17]. Para las pruebas de este sistema, el casco Meta II tiene una rotación inicial de $(0,0,0,1)$ con 0 en las componentes del vector del cuaternión $X$, $\mathrm{Y}, \mathrm{Z}$, y 1 para $\mathrm{W}$ que es la componente de rotación.

En el transcurso de la prueba, cuando el usuario con el casco Meta II puesto se ubica físicamente alrededor del cerebro virtual y decide una vía de acceso, se capturan los nuevos valores de posición y rotación que tiene el casco en ese momento.

En la figura 1 se puede observar la configuración inicial de la escena, donde la posición inicial del casco es $(0,0,0)$ y la del cerebro es $(0,-0.08,0.4)$. Estos valores de posición del cerebro se definen para que esté justo en frente de los ojos del usuario al iniciar la aplicación.

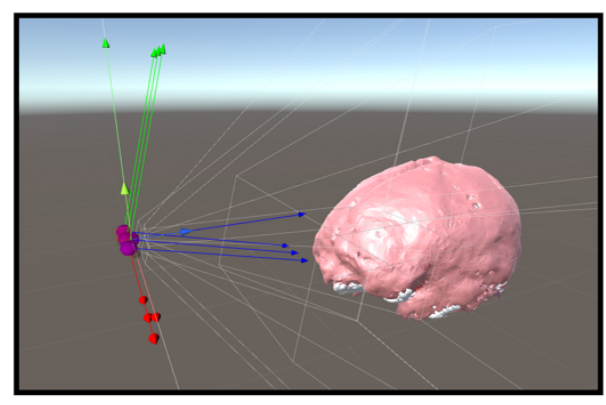

Figura 1. Configuración inicial de la escena

En la figura 2 se muestra un ejemplo de lo que usuario ve a través del Meta II cuando rota alrededor del cerebro para definir la vía de acceso.
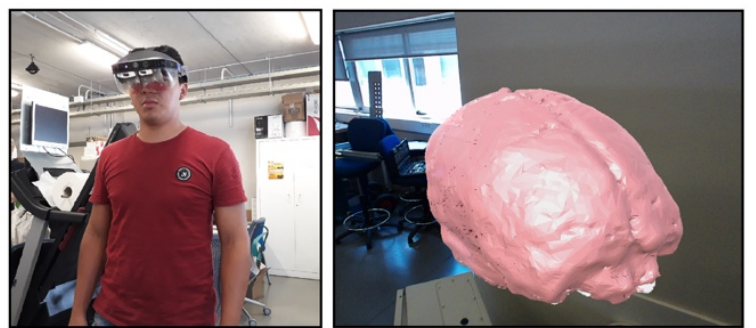

Figura 2. Visualización de la vía de acceso

\subsection{GENERACION DE MODELOS NEUROLÓGICOS A PARTIR DE IMAGEN MÉDICA}

\subsubsection{Modelos generados en 3D Slicer}

Para la generación de los modelos tridimensionales se utilizó 3D Slicer, el cual es una plataforma para análisis, visualización y procesamiento de imágenes médicas que permite generar modelos en $3 \mathrm{D}$ a partir de estas imágenes. Este software utiliza algoritmos para dicho procesamiento, entre los cuales en este proyecto se utilizaron Grow Cut para la segmentación de materia gris y blanca, Marching Cube que se utilizó para modelado de las superficies, y Streamline tractography para la representación de materia en tractos. [6].

Para representar el cerebro, inicialmente se obtuvo el modelo de la materia blanca y la materia gris a partir de las imágenes de resonancia magnética (MRI) de un paciente varón de 35 años. Para lograr lo anterior, primero se hizo la segmentación de dichas zonas en 10 imágenes consecutivas de la parte central de cada corte del cerebro (axial, sagital y coronal). Posterior a esto, se definen parámetros en los algoritmos anteriormente mencionados para generar los modelos tridimensionales.

En la figura 3 se puede observar los modelos obtenidos de la materia blanca y materia gris. 

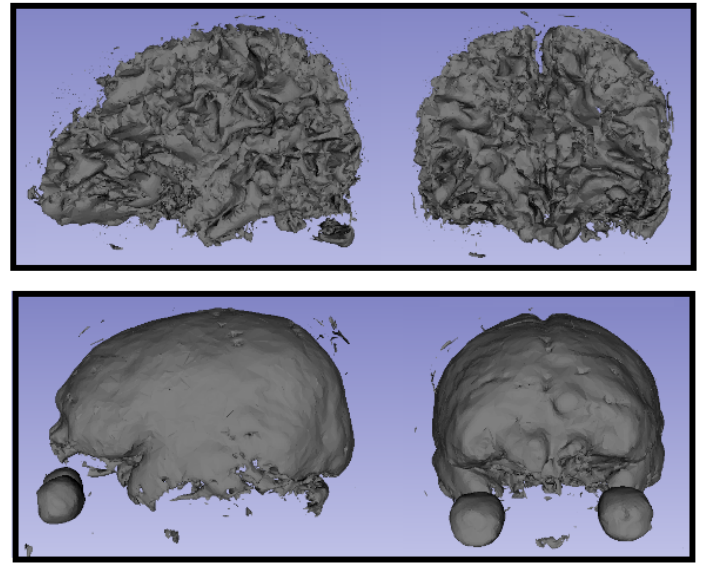

Figura 3. Materia blanca y materia gris generados a partir de MRI en 3D Slicer

De la misma manera, en las imágenes médicas se segmentaron 5 tumores ficticios en posiciones aleatorias, para obtener su modelo 3D mediante los algoritmos ya mencionados. La simulación de los tumores se hizo para fines prácticos ya que el paciente no presentaba ninguna patología.

Para representar el cuerpo calloso, se utilizó la extensión UKF tractography, la cual permite mediante su algoritmo de funcionamiento detectar la mayor cantidad de fibras posibles a partir de una región de interés. En este caso esa región se hace segmentando todo el cuerpo calloso en las mismas imágenes que se usaron para generar la materia gris y la materia blanca, y es a partir de ahí que se hace el seguimiento de las fibras del cuerpo calloso y se obtiene el modelo 3D que se muestra en la figura 4.
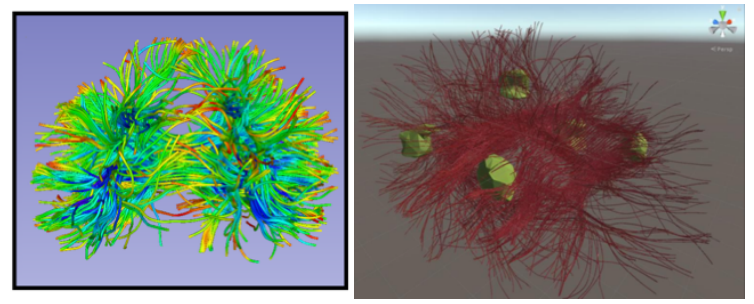

Figura 4. Tractografía del cuerpo calloso (Slicer) y tumores simulados (Unity)

\section{EXPERIMENTACIÓN RESULTADOS}

Para la validación de este sistema, se realizaron dos pruebas en la que participaron diez usuarios, donde se tuvo el cerebro virtual con cinco tumores que aparecerán de forma secuencial como escenario de realidad aumentada.

\subsection{DESCRIPCIÓN DEL EXPERIMENTO}

\subsubsection{Prueba 1: Cerebro fijo y usuario móvil}

Para esta prueba el cerebro estuvo en una posición fija durante el desarrollo de esta. Como primer paso, el usuario se coloca el casco Meta II, el cual ajusta y calibra a su medida. Luego, al ejecutar la aplicación el usuario debe físicamente sumergirse en el cerebro y navegar en él para buscar un primer tumor, y al encontrarlo debe posicionarse físicamente para decidir una posible vía de acceso para extirparlo, la cual comprometa la menor cantidad de tractos y materia blanca. Una vez hecho esto, se repite el procedimiento para los 4 tumores restantes. La prueba finaliza en el momento en el que el usuario defina la vía de acceso para el quinto tumor.

Para esta prueba se captura el tiempo que tardó el usuario en encontrar cada tumor, además se obtiene la posición física y los valores de la rotación del casco para cada una de las vías de acceso que definió el usuario para los cinco tumores.

\subsubsection{Prueba 2: Cerebro móvil y usuario fijo}

Para esta prueba se tiene el mismo escenario, con la diferencia que el cerebro se puede manipular con las manos y la cabeza del usuario debe estar fija. Una vez el usuario tenga puesto el casco Meta II ajustado y calibrado, se inicia la aplicación en la cual debe buscar los mismos tumores, de tal manera que solo pueda navegar en el cerebro virtual moviéndolo con una o dos manos y teniendo la cabeza fija. El sistema se diseñó para que el usuario pueda tocar, mover, rotar y variar el tamaño del cerebro virtual como lo desee. Cuando el usuario encuentra el primer tumor, debe manipular el cerebro para definir la vía de acceso con las mismas condiciones de la prueba 1. Finaliza la prueba cuando el usuario defina la vía de acceso para el tumor número 5.

En esta prueba se guardan los mismos datos de la primera prueba, además se obtiene la posición física y rotación final del cerebro cuando decide la vía de acceso para cada tumor.

En la figura 5 se observa a uno de los usuarios realizando la prueba 2 manipulando el cerebro con la mano cerrada representada con el circulo azul, y lo que está viendo a través del casco Meta II. 

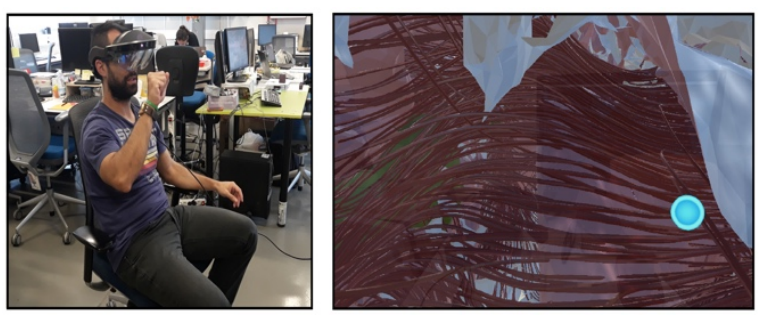

Figura 5. Usuario realizando la prueba 2

\subsection{RESULTADOS}

En la tabla 1 se observa la información correspondiente a los cinco tumores de las pruebas.

Tabla 1. Volumen y posición de los tumores

\begin{tabular}{|l|l|l|}
\hline Tumor & $\mathbf{V}\left(\mathbf{m m}^{3}\right)$ & Ubicación $(\mathbf{X}, \mathbf{Y}, \mathbf{Z})$ \\
\hline 1 & 556 & $(-38.73,-6.26,81.4)$ \\
\hline 2 & 500 & $(-5,21.4,-35.2)$ \\
\hline 3 & 480 & $(68.2,6.7,-12.6)$ \\
\hline 4 & 520 & $(-11.7,29.9,-135.5)$ \\
\hline 5 & 540 & $(6.5,0.13,-171.5)$ \\
\hline
\end{tabular}

En las figuras 6 y 7 se muestran los tiempos obtenidos por los usuarios para encontrar los cinco tumores en las dos pruebas.

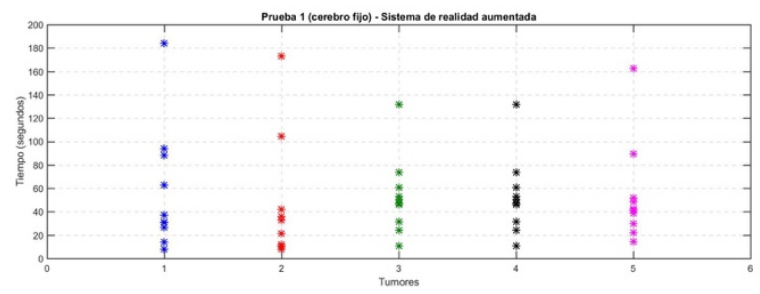

Figura 6. Tumores vs Tiempos para la prueba 1

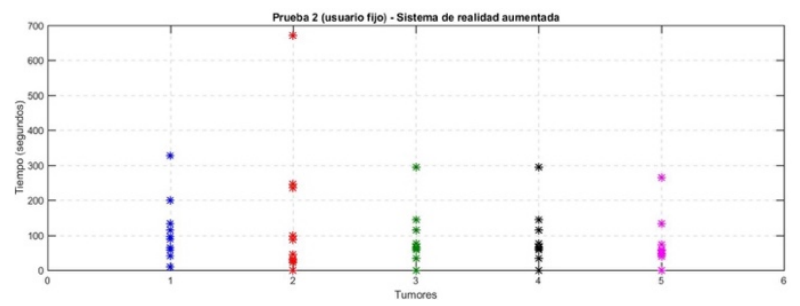

Figura 7. Tumores vs Tiempos para la prueba 2

\section{DISCUSIÓN}

\subsection{VALORACIÓN DE LOS RESULTADOS DE TIEMPO}

No se observó ningún comportamiento en la distribución de los tiempos entre los tumores en el experimento 1 , lo que parece indicar que no hay un aprendizaje durante el mismo en cuanto a las tareas de visualización, y los usuarios encuentran la misma dificultad en encontrar el tumor inicial que el tumor final. Sin embargo, en el experimento 2, en el que se debe manipular el objeto 3D, si se observa una ligera agrupación de tiempos a medida que se avanza la experimentación, lo que parece indicar que las tareas de manipulación del objeto virtual si son objeto de aprendizaje.

Por otra parte, se obtienen valores más altos de tiempos en el experimento 2, lo que indica que la necesidad de manipular el objeto añade una dificultad a la tarea de encontrar el tumor, la cual no existe si damos la posibilidad al usuario de desarrollarse dentro del entorno.

\subsection{VALORACIÓN DE LAS ENCUESTAS DE INMERSIÓN}

Al finalizar el experimento, cada usuario realizó una encuesta para la medición de inmersión en el sistema, dando como resultado una coincidencia de las valoraciones personales con lo observado en el análisis de los tiempos necesarios; una regularidad en las tareas de visualización y una dificultad (susceptible de mejorar con aprendizaje) en las tareas de manipulación.

Además, los resultados de las pruebas con 10 usuarios arrojaron que existe un gran interés en el uso de la tecnología la cual fue usable, absorbente y presentó un nivel de inmersión aceptable.

\section{Agradecimientos}

El presente trabajo se ha realizado dentro del acuerdo de colaboración entre la Universidad Miguel Hernández de Elche y la Universidad del Cauca, Colombia, y en el marco del proyecto DPI201680391-C3-2-R [AEI/FEDER, UE], financiado por la Agencia Estatal de Investigación (AEI) y por la Unión Europea a través del Fondo Europeo de Desarrollo Regional - FEDER - "Una manera de hacer Europa"

\section{English summary}

$\begin{array}{lcrr}\text { AUGMENTED } & \text { REALITY } & \text { IN } & \text { A } \\ \text { NAVIGATION } & \text { SYSTEM } & & \text { IN } \\ \text { NEUROSURGERY } & & & \end{array}$

Abstract

Minimally invasive surgery MIS, is a surgical technique that aims to reduce the damage to the tissues and organs. However, this technique has 
greatest challenge, overcoming visual limitations and a good capacity to create mental images of possible anatomical structures that are not visible. This paper presents a system prototype of neurosurgical navigation based on the augmented reality (AR) technique supported by the emerging technology of Head-Mounted Displays (HMD) or Immersive headmounted AR display to create a scenario that allows an extended visual sensation of neurological structures created by computer

to access anatomical structures obtained from real medical.

Two tests of the system were made with nine subjects, concluding that there is a great interest in the use of the technology, which was usable, engagement, absorbent and presented an acceptable level of immersion.

Keywords: Augmented reality, neurosurgery, surgical navigation.

\section{Referencias}

[1] Basogain, X., Olabe, M., Espinosa, K., Roueche, C., and Olabe, J., (2007) Realidad aumentada en la educación: una tecnología emergente, Escuela Superior de Ingeniería de Bilbao, EHU, Recuperado de http://bit.ly/2hpZokY

[2] Besharati Tabrizi, L., and Mahvash, M., (2015) "Augmented reality-guided neurosurgery: accuracy and intraoperative application of an image projection technique". Journal of neurosurgery, pp. 206-211.

[3] Bittermann, G., Scheifele, C., Prokic, V., Bhatt, V., Henke, M., Grosu, A., Schmelzeisen, R., and Metzger, M. C, (2013) Description of a method: computer generated virtual model for accurate localisation of tumour margins, standardised resection, and planning of radiation treatment in head \& neck cancer surgery, Journal of CranioMaxillo-Facial Surgery, pp. 279-281.

[4] Centella-Gutierrez, C., and Dean-Ferrer, A, (2016) Aportaciones de la cirugía guiada por ordenador y navegación en oncología de cabeza y cuello: una revisión bibliográfica sistemática y actualización, Revista Española de Cirugía Oral y Maxilofacial, pp. 136-142.

[5] Favieri, A., (2008) Introducción a los Cuaterniones. Facultad Regional Haedo, Universidad Tecnológica Nacional, Argentina: Editorial de la Universidad Tecnológica Nacional.
[6] Fedorov, A., Beichel, R., Kalpathy-Cramer, J., Finet, J., Fillion-Robin, J.-C., Pujol, S., ... Kikinis, R. (2012) 3D Slicer as an Image Computing Platform for the Quantitative Imaging Network. Magnetic Resonance Imaging, 30 (9), 1323-1341. Recuperado de http://doi.org/10.1016/j.mri.2012.05.001

[7] S. L. Kim, H. J. Suk, J. H. Kang, J. M. Jung, T. H. Laine and J. Westlin, (2014) "Using Unity 3D to facilitate mobile augmented reality game development," In Internet of Things (WF-IoT), World Forum, IEEE, pp. 21-26.

[8] Nicolau, S., Soler, L., Mutter, D., and Marescaux, J., (2011) Augmented Reality in laparoscopic surgical oncology. Surgical oncology, pp. 189-201.

[9] Noreña, T., and Romero, E., (2013) Compresión de imágenes médicas. Biomédica, 33 (1), pp. 137-151.

[10] Ortiz Rangel, C. E., (2011) “Augmented reality in medicine". Revista Colombiana de Cardiología, pp.4-7.

[11] Pelargos, P. E., Nagasawa, D. T., Lagman, C., Tenn, S., Demos, J. V., Lee, S. J., Bui, T. T., Barnette, N. E., Bhatt, N. S., Ung, N., Bari, A., Martin, N., Yang, I. (2017) "Utilizing virtual and augmented reality for educational and clinical enhancements in neurosurgery". Journal of Clinical Neuroscience, pp.1-4.

[12] Roncagliolo, P., (2014) Desarrollo de un sistema de realidad aumentada para entrenamiento en planificación neuroquirúrgica. (Tesis de maestría). Universidad técnica Federico Santa María, Valparaíso, Chile.

[13] Torres del Castillo, G., (1999) Representing rotations by quaternions. Miscelánea matemática, Universidad Autónoma de Puebla, México.

[14] Velásquez, O., Matar, O. A., and Jaimes, F. (2018) Tratamiento quirúrgico de la endocarditis mediante cirugía mínimamente invasiva. Revista Colombiana de Cardiología.

[15] Vávra, P., Roman, J., Zonča, P., Ihnát, P., Němec, M., Kumar, J., Habib, H., and El-Gendi, A. (2017) Recent Development of Augmented Reality in Surgery: A Review. Journal of Healthcare Engineering. Recuperado de http://doi.org/10.1155/2017/4574172 
[16] Malcolm, J. G., Shenton, M. E., \& Rathi, Y. (2009) Neural Tractography Using An Unscented Kalman Filter. Information Processing in Medical Imaging: Proceedings of the Conference, 21, pp. 126-138.

[17] Hanson, A. J., (2005) Visualizing quaternions, ACM SIGGRAPH 2005 Courses, ACM, New York, USA

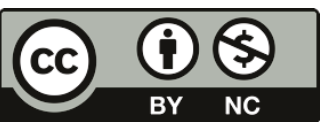

(C) 2018 by the authors. Submitted for possible open access publication under the terms and conditions of the Creative Commons Attribution CC-BY-NC 3.0 license (https://creativecommons.org/licenses/by-nc/3.0). 